\title{
Spectral Analysis of Certain Schrödinger Operators
}

\author{
Mourad E.H. ISMAIL ${ }^{\dagger}$ and Erik KOELINK $\ddagger$ \\ $\dagger$ Department of Mathematics, University of Central Florida, Orlando, FL 32816, USA \\ E-mail: mourad.ismail@ucf.edu \\ URL: http://www.math.ucf.edu/ ismail/ \\ $\ddagger$ Radboud Universiteit, IMAPP, FNWI, Heyendaalseweg 135, 6525 AJ Nijmegen, \\ the Netherlands \\ E-mail: e.koelink@math.ru.nl \\ URL: http://www.math.ru.nl/ koelink/
}

Received May 07, 2012, in final form September 12, 2012; Published online September 15, 2012

http://dx.doi.org/10.3842/SIGMA.2012.061

\begin{abstract}
The $J$-matrix method is extended to difference and $q$-difference operators and is applied to several explicit differential, difference, $q$-difference and second order AskeyWilson type operators. The spectrum and the spectral measures are discussed in each case and the corresponding eigenfunction expansion is written down explicitly in most cases. In some cases we encounter new orthogonal polynomials with explicit three term recurrence relations where nothing is known about their explicit representations or orthogonality measures. Each model we analyze is a discrete quantum mechanical model in the sense of Odake and Sasaki [J. Phys. A: Math. Theor. 44 (2011), 353001, 47 pages].

Key words: $J$-matrix method; discrete quantum mechanics; diagonalization; tridiagonalization; Laguere polynomials; Meixner polynomials; ultraspherical polynomials; continuous dual Hahn polynomials; ultraspherical (Gegenbauer) polynomials; Al-Salam-Chihara polynomials; birth and death process polynomials; shape invariance; zeros
\end{abstract}

2010 Mathematics Subject Classification: 30E05; 33C45; 39A10; 42C05; 44A60

\section{Introduction}

The $J$-matrix method started with the pioneering works of Yamani, Heller and Reinhardt [19, 20, 40] in the early 1970's and has been applied by Yamani, Heller and Reinhardt to different physical models. Some of the recent applications of the $J$-matrix method to physics are spearheaded by Alhaidari and his research team, [2, 3, 4, 5, 7]. The $J$-matrix principle says that the spectrum of a tridigonalizable operator is the same as the tridiagonal matrix representing it. Such a tridiagonal matrix can be split into irreducible blocks, and to each of these blocks there is a corresponding set of orthogonal polynomials. Moreover, the eigenfunctions of a tridiagonalizable operator can be expressed using these corresponding polynomials, and in the self-adjoint case the spectral measure is related to the orthogonality measures of the orthogonal polynomials. This general set-up is described and proved in [24] where we considered the Schrödinger equation with the Morse potential as an example. Our later work [23] develops a general scheme for tridiagonalizing differential, difference, or $q$-difference operators arising from two sets of related orthogonal polynomials. In particular, we find in [23] the Jacobi transform and its special case the Mehler-Fock transform originally introduced by Mehler to study electrical distributions.

Tridiagonalization of an explicit symmetric or self-adjoint operator $T$, like a differential or $(q-)$ difference operator on an explicit Hilbert space $\mathcal{H}$ of functions, amounts to finding an explicit 
orthonormal basis $\left\{e_{n}\right\}_{n=0}^{\infty}$ so that we can realize

$$
T e_{n}=\alpha_{n} e_{n+1}+\beta_{n} e_{n}+\alpha_{n-1} e_{n}, \quad \alpha_{n}>0, \quad \beta_{n} \in \mathbb{R},
$$

with respect to this basis. Since three-term operators, or Jacobi operators, can be described in terms of orthogonal polynomials, we can obtain information on $T$ in terms of properties of the orthogonal polynomials, see [24] and references given there. Assuming that there are orthgonal polynomials $p_{n}$ (corresponding to a determinate moment problem) satisfying

$$
\lambda p_{n}(\lambda)=\alpha_{n} p_{n+1}(\lambda)+\beta_{n} p_{n}(\lambda)+\alpha_{n-1} p_{n}(\lambda), \quad \int_{\mathbb{R}} p_{n}(\lambda) p_{m}(\lambda) d \mu(\lambda)=\delta_{n m},
$$

then the spectral decomposition is $U T=M U$, where $U: \mathcal{H} \rightarrow L_{2}(\mu), e_{n} \mapsto p_{n}$ and $M: L_{2}(\mu) \rightarrow$ $L_{2}(\mu)$ is the multiplication operator. The link between more general differential, difference or $q-$ difference operators, and Jacobi matrices can be very useful to study the spectral decomposition of the original operator in terms of the orthogonal polynomials and vice versa. So we can obtain information on one of the operators by transferring information from the other, and we show this in particular examples in this paper. In particular, in case information on both operators is known, we obtain even more explicit results, and examples of this approach can be found in [23]. However, it is not straightforward to find explicit tridiagonalization of a given operator, and here we present ways to obtain operators with a tridiagonalization. In the tridiagonalization of the operators in this paper it is often the case that the polynomials cannot be matched directly with known polynomials [21, 29], and in these cases we have given some information on the support of the spectral meaure of these polynomials. A treatment of the spectral theory of differential operators can be found in many sources, and we refer the interested reader to the excellent book by Edmunds and Evans [15]. The spectral theory of tridiagonal matrices and their connection with orthogonal polynomials and the moment problem is in $[1,14,30,38]$.

The contents of the paper are as follows. In Section 2 we record the formulas used in the sequel. The basic references are $[8,16,17,21,29,37]$. The expert reader may easily skip Section 2 . In Sections 3 and 4 we start with an operator with known orthogonal polynomial eigenfunctions then multiply it by a linear function of the space variable and study the spectral properties of the new Hamiltonian. Section 3 treats the case of Laguerre polynomials, leading to tridiagonalization involving continuous dual Hahn polynomials. It is simple enough but contains all the ingredients of the method. Section 4 treats the Meixner polynomials and the $J$-matrix method leads to a one parameter generalization of the continuous dual Hahn polynomials. The examples in Sections 3 and 4 are related to the approach of [23]. In Section 5 we introduce a different modification. We start with an operator $T$ which is diagonalized by a known polynomial system. We then consider the Schödinger operator $T+\gamma x$. Such an equation arises for example in the case of a charged particle in the presence of a uniform electric field. In this case $\gamma=-q F$, where $F$ is the magnitude of the electric field and $q$ is the electric charge, see [32, § 24], or [10].

The Schödinger operator $T+\gamma x$ is automatically tridiagonal in the orthogonal polynomial basis which diagonalizes or tridiagonalizes $T$. We study the spectral properties of $T+\gamma x$ for four different sets of polynomials. In Section 6 we combine both generalizations of Sections 3 and 4 for the case of the Laguerre and Meixner polynomials. Finally, in Section 7 we study this approach for two families of $q$-orthogonal polynomials, namely the Al-Salam-Chihara polynomials and the $q^{-1}$-Hermite polynomials. The $q^{-1}$-Hermite polynomials correspond to an indeterminate moment problem, so we study the corresponding $q$-difference operator on the weighted $L^{2}$-space corresponding to a $N$-extremal measure. It turns out that the polynomials in the tridiagonalization is again corresponding to an indeterminate moment problem, so that the $q$-difference operator is not essentially self-adjoint on the space of polynomials.

We end by noting that more differential, difference and $q$-difference operators can be studied using the $J$-matrix method. In particular, we can study classes of higher-order operators in this way as well. 


\section{Preliminaries}

In this section we recall some results needed in the sequel. We first record the properties of the Laguerre polynomials. They satisfy the differential relations, [21, (4.6.13), (4.6.15)]:

$$
\begin{aligned}
& \frac{d}{d x} L_{n}^{(\alpha)}(x)=-L_{n-1}^{(\alpha+1)}(x) \\
& \frac{1}{x^{\alpha} e^{-x}} \frac{d}{d x}\left[x^{\alpha+1} e^{-x} \frac{d}{d x} L_{n}^{(\alpha)}(x)\right]=-n L_{n}^{(\alpha)}(x) .
\end{aligned}
$$

A generating function of the Laguerre polynomials is

$$
\sum_{n=0}^{\infty} L_{n}^{(\alpha)}(x) t^{n}=(1-t)^{-\alpha-1} \exp \left(\frac{-x t}{1-t}\right),
$$

see $[21,(4.6 .4)],[29,37]$ and it implies

$$
L_{n}^{(\alpha)}(x)=L_{n}^{(\alpha+1)}(x)-L_{n-1}^{(\alpha+1)}(x) .
$$

The orthogonality relation is

$$
\int_{0}^{\infty} x^{\alpha} e^{-x} L_{m}^{(\alpha)}(x) L_{n}^{(\alpha)}(x) d x=\frac{\Gamma(\alpha+n+1)}{n !} \delta_{m, n}, \quad \alpha>-1 .
$$

The Meixner polynomials are, $[21, \S 6.1],[29, \S 1.9]$,

$$
M_{n}(x ; \beta, c)={ }_{2} F_{1}(-n,-x ; \beta ; 1-1 / c),
$$

and have the generating function

$$
\sum_{n=0}^{\infty} \frac{(\beta)_{n}}{n !} M_{n}(x ; \beta, c) t^{n}=(1-t / c)^{x}(1-t)^{-x-\beta} .
$$

The orthogonality relation is

$$
\sum_{x=0}^{\infty} M_{m}(x ; \beta, c) M_{n}(x ; \beta, c) \frac{(\beta)_{x}}{x !} c^{x}=\frac{c^{-n} n !}{(\beta)_{n}(1-c)^{\beta}} \delta_{m, n},
$$

valid for $\beta>0,0<c<1$ and their three term recurrence relation is

$$
\begin{aligned}
-x M_{n}(x ; \beta, c)= & \frac{c(\beta+n)}{1-c} M_{n+1}(x ; \beta, c)+\frac{n}{1-c} M_{n-1}(x ; \beta, c) \\
& -\frac{n+c(\beta+n)}{1-c} M_{n}(x ; \beta, c) .
\end{aligned}
$$

The Meixner polynomials satisfy the second order difference equation

$$
\frac{x !}{c^{x}(\beta)_{x}} \nabla\left(\frac{(\beta+1)_{x} c^{x}}{x !} \Delta\right) M_{n}(x ; \beta, c)=\frac{n}{\beta} \frac{c-1}{c} M_{n}(x ; \beta, c),
$$

where

$$
(\Delta f)(x)=f(x+1)-f(x), \quad(\nabla f)(x)=f(x)-f(x-1) .
$$


The Meixner-Pollaczek polynomials $\left\{P_{n}^{(\lambda)}(x ; \phi)\right\}$ satisfy the orthogonality relation [29, (1.7.2)]

$$
\frac{1}{2 \pi} \int_{\mathbb{R}} e^{(2 \phi-\pi) x}|\Gamma(\lambda+i x)|^{2} P_{m}^{(\lambda)}(x ; \phi) P_{n}^{(\lambda)}(x ; \phi) d x=\frac{\Gamma(n+2 \lambda)}{(2 \sin \phi)^{2 \lambda} n !} \delta_{m, n},
$$

for $\lambda>0,0<\phi<\pi$, and the three term recurrence relation [29, (1.7.3)]

$$
(n+1) P_{n+1}^{(\lambda)}(x ; \phi)+(n+2 \lambda-1) P_{n-1}^{(\lambda)}(x ; \phi)=2[x \sin \phi+(n+\lambda) \cos \phi] P_{n}^{(\lambda)}(x ; \phi),
$$

with $P_{0}^{(\lambda)}(x ; \phi)=1, P_{1}^{(\lambda)}(x ; \phi)=2[x \sin \phi+\lambda \cos \phi]$.

We parametrize the independent variable $x$ by $x=(z+1 / z) / 2$ and given a function we set $\breve{f}(z)=f(x)$. The Askey-Wilson operator $\mathcal{D}_{q}$ and the averaging operator $\mathcal{A}_{q}$ are defined by, [21, $\S 12.1]$,

$$
\left(\mathcal{D}_{q} f\right)(x)=\frac{\breve{f}\left(z q^{1 / 2}\right)-\breve{f}\left(z q^{-1 / 2}\right)}{\breve{e}\left(q^{1 / 2} z\right)-\breve{e}\left(q^{-1 / 2} z\right)}, \quad\left(\mathcal{A}_{q} f\right)(x)=\frac{1}{2}\left[\breve{f}\left(z q^{1 / 2}\right)+\breve{f}\left(z q^{-1 / 2}\right)\right],
$$

where $e(x)=x=(z+1 / z) / 2$.

The Askey-Wilson operator is well-defined on $H_{1 / 2}$, where

$$
H_{\nu}:=\left\{f: f((z+1 / z) / 2) \text { is analytic for } q^{\nu} \leq|z| \leq q^{-\nu}\right\} .
$$

Let $\mathcal{H}_{w}$ denote the weighted space $L^{2}(-1,1 ; w(x) d x)$ with inner product

$$
(f, g)_{w}:=\int_{-1}^{1} f(x) \overline{g(x)} w(x) d x, \quad\|f\|_{w}:=(f, f)_{w}^{1 / 2}
$$

and let $T$ be defined by

$$
T f(x):=-\frac{1}{w(x)} \mathcal{D}_{q}\left(p \mathcal{D}_{q} f\right)(x),
$$

for $f$ in $H_{1}$. We shall assume that $p$ and $w$ are positive on $(-1,1)$ and also satisfy

(i) $p(x) / \sqrt{1-x^{2}} \in H_{1 / 2}, 1 / p \in L(-1,1)$,

(ii) $w(x) \in L(-1,1), 1 / w \in L\left(-1,1 ; \frac{d x}{\left(1-x^{2}\right)}\right)$.

The expression $T f$ is therefore defined for $f \in H_{1}$, and the operator $T$ acts in $\mathcal{H}_{w}$. Furthermore, the domain $H_{1}$ of $T$ is dense in $\mathcal{H}_{w}$ since it contains all polynomials. The following theorem is due to Brown, Evans and Ismail [12].

Theorem 2.1. The operator $T$ is symmetric in $\mathcal{H}_{w}$ and positive.

The Al-Salam-Chihara polynomials are defined by [21, (15.1.6)]

$$
p_{n}\left(x ; t_{1}, t_{2} \mid q\right)={ }_{3} \phi_{2}\left(\begin{array}{c}
q^{-n}, t_{1} e^{i \theta}, t_{1} e^{-i \theta} \\
t_{1} t_{2}, 0
\end{array} \mid q, q\right) .
$$

Their weight function is

$$
w\left(\cos \theta ; t_{1}, t_{2}\right):=\frac{\left(e^{2 i \theta}, e^{-2 i \theta} ; q\right)_{\infty} / \sin \theta}{\left(t_{1} e^{i \theta}, t_{1} e^{-i \theta}, t_{2} e^{i \theta}, t_{2} e^{-i \theta} ; q\right)_{\infty}},
$$

and their orthogonality relation will be stated in (7.4). The generating function for the AlSalam-Chihara polynomials is [21, (15.1.10)]

$$
\sum_{n=0}^{\infty} \frac{\left(t_{1} t_{2} ; q\right)_{n}}{(q ; q)_{n}}\left(\frac{t}{t_{1}}\right)^{n} p_{n}\left(\cos \theta ; t_{1}, t_{2}\right)=\frac{\left(t t_{1}, t t_{2} ; q\right)_{\infty}}{\left(t_{1} e^{i \theta}, t_{1} e^{-i \theta} ; q\right)_{\infty}} .
$$


Theorem 2.2. Consider the three term recurrence relation in orthonormal form

$$
x p_{n}(x)=a_{n+1} p_{n+1}(x)+b_{n} p_{n}(x)+a_{n} p_{n-1}(x), \quad n \geq 0, \quad a_{n}>0, \quad b_{n} \in \mathbb{R},
$$

with $a_{0} p_{-1}(x):=0$. Then the moment problem is determinate, that is, it has a unique solution, if one of the following conditions hold

$$
\begin{array}{ll}
\sum_{n=0}^{\infty} \frac{\left|b_{n+1}\right|}{a_{n+1} a_{n+2}}=\infty, & \\
a_{n}+b_{n}+a_{n+1} \leq C, & \text { for some } C, \\
a_{n}-b_{n}+a_{n+1} \leq C, & \text { for some } C .
\end{array}
$$

The condition (2.12) is Exercise 2 on p. 25 of [1], while (2.13), (2.14) are Theorem VII.1.4 and its corollary in [11, pp. 505-506].

Theorem 2.3. Let $p_{n}(x)$ be generated by (2.11). Then the zeros of the polynomial $p_{n}(x)$ are in $(A, B)$, where

$$
B=\max \left\{x_{j}: 0<j<n\right\}, \quad A=\min \left\{y_{j}: 0<j<n\right\},
$$

where $y_{j} \leq x_{j}$ and

$$
x_{j}, y_{j}=\frac{1}{2}\left(b_{j}+b_{j-1}\right) \pm \frac{1}{2} \sqrt{\left(b_{j}-b_{j-1}\right)^{2}+16 a_{j}^{2}}, \quad 1 \leq j<n .
$$

Theorem 2.3 is the special case $c_{n}=1 / 4$ of a result due to Ismail and $\mathrm{Li}$ in [26]. The full result is also stated and proved in [21, Theorem 7.2.7].

The zeros of orthogonal polynomials are real and simple, so we shall follow the standard notation in [39] or [21] and arrange the zeros $x_{n, j}, 1 \leq j \leq n$ as

$$
x_{n, 1}>x_{n, 2}>\cdots>x_{n, n}
$$

\section{A differential operator related to the Laguerre polynomials}

Consider the differential operator

$$
\left(T_{L} f\right)(x)=\frac{1}{x^{\alpha} e^{-x}} \frac{d}{d x}\left[x^{\alpha+2} e^{-x} \frac{d f}{d x}\right] .
$$

We will discuss a generalization of this operator in Section 6 . The boundary value problem we are interested in is $T_{L} y=\lambda y$ with the boundary conditions $x^{1+\alpha / 2} f(x) e^{-x / 2} \rightarrow 0$ as $x \rightarrow 0$ and $x \rightarrow \infty$. The equation $T_{L} y=\lambda y$ is

$$
x^{2} y^{\prime \prime}+(\alpha+2) x y^{\prime}-x^{2} y^{\prime}=\lambda y \text {. }
$$

It is easy to see that $T_{L}$ is symmetric on weighted $L_{2}$ space with the inner product

$$
(f, g)=\int_{0}^{\infty} x^{\alpha} e^{-x} f(x) \overline{g(x)} d x .
$$


The $(m, n)$ matrix elements of $T_{L}$ as an operator in $L_{2}\left(0, \infty, x^{\alpha} e^{-x}\right)$ in the basis $\left\{L_{n}^{(\alpha)}(x)\right\}$ can be calculated using (2.3), (2.4), (2.2);

$$
\begin{aligned}
& \left(T_{L} L_{n}^{(\alpha)}, L_{m}^{(\alpha)}\right)=\int_{0}^{\infty} L_{m}^{(\alpha)}(x) \frac{d}{d x}\left[x^{\alpha+2} e^{-x} \frac{d}{d x} L_{n}^{(\alpha)}(x)\right] d x \\
& =\int_{0}^{\infty}\left[L_{m}^{(\alpha+1)}(x)-L_{m-1}^{(\alpha+1)}(x)\right] \frac{d}{d x}\left[x^{\alpha+2} e^{-x} \frac{d}{d x}\left(L_{n}^{(\alpha+1)}(x)-L_{n-1}^{(\alpha+1)}(x)\right)\right] d x \\
& =-\int_{0}^{\infty} x^{\alpha+1} e^{-x}\left[L_{m}^{(\alpha+1)}(x)-L_{m-1}^{(\alpha+1)}(x)\right]\left[n L_{n}^{(\alpha+1)}(x)-(n-1) L_{n-1}^{(\alpha+1)}(x)\right] d x \\
& =-\frac{\Gamma(n+\alpha+2)}{(n-1) !} \delta_{m, n}+\frac{\Gamma(m+\alpha+2)}{(m-1) !} \delta_{m+1, n}+\frac{\Gamma(n+\alpha+2)}{(n-1) !} \delta_{m, n+1}-\frac{\Gamma(n+\alpha+1)}{(n-2) !} \delta_{m, n} \\
& =\frac{\Gamma(m+\alpha+2)}{(m-1) !} \delta_{m+1, n}-(\alpha+2 m) \frac{\Gamma(n+\alpha+1)}{(m-1) !} \delta_{m, n}+\frac{\Gamma(m+\alpha+1)}{(m-2) !} \delta_{m, n+1} .
\end{aligned}
$$

Thus the sought matrix representation of $T_{L}$ is tridiagonal. It is also clear the constants are in the null space of $T_{L}$, so we mod out by the constant functions. Let $\left\{A_{m, n}(L)\right\}$ be the matrix elements. Thus $A_{m, n}(L)$ is $\sqrt{\frac{m ! n !}{\Gamma(m+\alpha+1) \Gamma(n+\alpha+1)}}$ times the above expression. Thus

$$
\begin{aligned}
A_{m, n}(L)= & m \sqrt{(m+1)(m+\alpha+1)} \delta_{m+1, n} \\
& -m(2 m+\alpha) \delta_{m, n}+(m-1) \sqrt{m(\alpha+m)} \delta_{m, n+1} .
\end{aligned}
$$

The effect of modding out by the constants is to delete the first row and column of the matrix is to shift $m$ and $n$ by one. Thus we consider the tridiagonal matrix $B=\left(B_{m, n}\right), m, n=0,1, \ldots$,

$$
\begin{aligned}
B_{m, n}= & (m+1) \sqrt{(m+2)(m+\alpha+2)} \delta_{m, n-1} \\
& -(m+1)(2 m+\alpha+2) \delta_{m, n}+m \sqrt{(m+1)(\alpha+m+1)} \delta_{m, n+1} .
\end{aligned}
$$

Now the spectral equation $E X=B X$ where $X$ is a column vector, when written componentwise is a three term recurrence relations and the component of $X$ are $p_{n}(E)$. The corresponding monic polynomials satisfy the three term recurrence relation

$$
E p_{m}(E)=p_{m+1}(E)-(m+1)(2 m+\alpha+2) p_{m}(E)+m^{2}(m+1)(m+\alpha+1) p_{m-1}(E) .
$$

This is $[29,(1.3 .5)]$ and identifies the $p_{m}$ 's as continuous dual Hahn polynomials with the parameter and variable identifications

$$
a=\frac{1-\alpha}{2}, \quad b=\frac{1+\alpha}{2}, \quad c=\frac{3+\alpha}{2}, \quad E=-x-\frac{(\alpha+1)^{2}}{4} .
$$

As stated in the Introduction the spectral decomposition is given in terms of the orthogonality measure for the continuous dual Hahn polynomials $S_{n}(x ; a, b, c)$, see $[29, \S 1.3]$. The measure is absolutely continuous on $[0, \infty)$ in case $a, b$ and $c$ are positive. In case one of them is negative, finitely many discrete mass points have to be added. In the present case however we assumed $\alpha>-1$, hence only $a$ can be negative. Explicitly, with $S_{n}(x)=S_{n}(x ; a, b, c)$,

$$
\begin{aligned}
& \frac{1}{2 \pi \Gamma(a+b) \Gamma(a+c) \Gamma(b+c)} \int_{0}^{\infty} S_{n}\left(y^{2}\right) S_{m}\left(y^{2}\right)\left|\frac{\Gamma(a+i y) \Gamma(b+i y) \Gamma(c+i y)}{\Gamma(2 i y)}\right|^{2} d y \\
& +\frac{\Gamma(b-a) \Gamma(c-a)}{\Gamma(-2 a) \Gamma(b+c)} \sum_{k=0}^{M} S_{n}\left(-(a+k)^{2}\right) S_{m}\left(-(a+k)^{2}\right) \frac{(2 a)_{k}(a+1)_{k}(a+b)_{k}(a+c)_{k}}{k !(a)_{k}(a-b+1)_{k}(a-c+1)_{k}}(-1)^{k} \\
& =\delta_{n m} n !(a+b)_{n}(a+c)_{n}(b+c)_{n},
\end{aligned}
$$

where $M=\max \{k \in \mathbb{N}: k+a<0\}$.

Now [24, Theorem 2.7] gives the following proposition. 
Proposition 3.1. The unbounded operator $T_{L}$ acting on the subspace of polynomials in $L_{2}\left(0, \infty, x^{\alpha} e^{-x}\right)$ is essentially self-adjoint. The spectrum of the closure has an absolutely continuous part $\left(-\infty,-\frac{1}{4}(\alpha+1)^{2}\right]$. The discrete spectrum consists of $\{0\}$ and $\left\{E_{k} \mid k \in\{0, \ldots, M\}\right\}$, where $M=\max \{k \in \mathbb{N} \mid k+(1-\alpha) / 2<0\}$ and $E_{k}=(k+1)(k-\alpha)$.

The explicit spectral measure can be obtained from the orthogonality measure for the continuous dual Hahn polynomials (3.4), cf. Section 1.

This discussion of the differential operator $T_{L}$ is related to the set-up of [23], where the case related to Jacobi polynomials is considered. In [23] we assume that we did not need to mod out a null space. The differential operator $T_{L}$ can be related to the confluent hypergeometric differential equation in the same way as the hypergeometric differential operator shows up in $[23, \S 3]$.

\section{A difference operator related to Meixner polynomials}

The generating function (2.5) implies

$$
\beta M_{n}(x ; \beta, c)=(\beta+n) M_{n}(x ; \beta+1, c)-n M_{n-1}(x ; \beta+1, c) .
$$

The second order linear operator to be considered is $T_{M}$,

$$
\left(T_{M} f\right)(x):=\frac{x !}{c^{x}(\beta)_{x}} \nabla\left(\frac{(\beta+2)_{x} c^{x}}{x !} \Delta f\right)(x) .
$$

We consider the inner product spaces endowed with the inner product

$$
\langle f, g\rangle_{\beta}=\sum_{x=0}^{\infty} \frac{c^{x}(\beta)_{x}}{x !} f(x) \overline{g(x)} .
$$

The operator $T_{M}$ is formally self-adjoint with respect to $\langle\cdot, \cdot\rangle_{\beta}$.

Using (4.1) and (2.7) we see that

$$
T_{M} M_{n}(x ; \beta, c)=\frac{(\beta+x)(c-1)}{\beta^{2} c(\beta+1)}\left[n(\beta+n) M_{n}(x ; \beta+1, c)-n(n-1) M_{n-1}(x ; \beta+1, c)\right] .
$$

Therefore

$$
\begin{aligned}
& \beta^{2} \frac{c(\beta+1)}{(c-1)}\left\langle M_{m}(x ; \beta, c), T_{M} M_{n}(x ; \beta, c)\right\rangle_{\beta} \\
& =\left\langle(\beta+m) M_{m}(x ; \beta+1, c)-m M_{m-1}(x ; \beta+1, c),\right. \\
& \left.\quad n(\beta+n) M_{n}(x ; \beta+1, c)-n(n-1) M_{n-1}(x ; \beta+1, c)\right\rangle_{\beta+1} \\
& =m(\beta+m)^{2} h_{m}(\beta+1) \delta_{m, n}-m(m+1)(\beta+m) h_{m}(\beta+1) \delta_{m, n-1} \\
& \quad-m(m-1)(\beta+m-1) h_{m-1}(\beta+1) \delta_{m, n+1}+m^{2}(m-1) h_{m-1}(\beta+1) \delta_{m, n},
\end{aligned}
$$

where

$$
h_{n}(\beta)=\left\langle M_{n}(x ; \beta, c), M_{n}(x ; \beta, c)\right\rangle_{\beta}=\frac{c^{-n} n !}{(\beta)_{n}(1-c)^{\beta}} .
$$

Since $T_{M}$ annihilates constants we mod out by the space of constants and let the matrix elements of $T_{M}$ be $\left\{B_{m, n}: m, n \geq 0\right\}$. Thus

$$
B_{m, n}(M)=\frac{\left\langle M_{m+1}(x ; \beta, c), T_{M} M_{n+1}(x ; \beta, c)\right\rangle_{\beta}}{\sqrt{h_{m+1}(\beta) h_{n+1}(\beta)}} .
$$


In other words

$$
\begin{aligned}
c \beta(\beta+1) B_{m, n}= & -[(m+1)(m+\beta+1)+m(m+1) c] \delta_{m, n} \\
& +m \sqrt{c(m+1)(m+\beta)} \delta_{m, n+1}+(m+1) \sqrt{c(m+2)(\beta+m+1)} \delta_{m, n-1} .
\end{aligned}
$$

Now scale the energy parameter $E$ by $E=-x /(c \beta(\beta+1))$. This translates into the monic three term recurrence relation

$$
\begin{aligned}
x P_{m}(x)= & P_{m+1}(x)+[(m+1)(m+\beta+1)+m(m+1) c] P_{m}(x) \\
& +c m^{2}(m+1)(\beta+m) P_{m-1}(x) .
\end{aligned}
$$

The polynomials generated by (4.5) seem to be new. They give a one parameter generalization of the continuous dual Hahn polynomials which is different from the Wilson polynomials. Finding the orthogonality measure of these polynomials remains a challenge. It clear that the measure is unique and is supported on an unbounded subset of $[0, \infty)$. They are birth and death process polynomials corresponding to birth rates $b_{n}=(n+1)(\beta+n+1)$ and death rates $d_{n}=c n(n+1)$, see (5.6). By (2.14) the corresponding moment problem is determinate. So in this case we do not have a precise analogue of Proposition 3.1, except that the unbounded operator $T_{M}$ defined on the polynomials has a unique self-adjoint extension.

\section{Operators with additional potential}

We consider the case of second order operators which arise from classical orthogonal polynomials. Let $p_{n}(x)$ be a monic family of classical orthogonal polynomials and $T$ a second order operator such that

$$
T p_{n}(x)=\lambda_{n} p_{n}(x) .
$$

Also assume that the three term recurrence relation for the $p_{n}$ 's is

$$
x p_{n}(x)=p_{n+1}(x)+\alpha_{n} p_{n}(x)+\beta_{n} p_{n-1}(x) .
$$

We now consider the spectral problem

$$
(T+\gamma x) \psi(x, E)=E \psi(x, E) .
$$

One can think of $T y=E y$ as a free particle problem then (5.3) will be a Schrödinger problem with potential $\gamma x$. Let $\mu$ be the orthogonality measure of $\left\{p_{n}(x)\right\}$ and assume we deal with the case when the polynomials are dense in $L_{2}(\mathbb{R}, \mu)$, which is true with very few exceptions. The

orthonormal polynomials are $\left\{p_{n}(x) / \sqrt{\beta_{1} \beta_{2} \cdots \beta_{n}}\right\}$ and form a basis for $L_{2}(\mathbb{R}, \mu)$. The matrix element of $T+\gamma x$ with respect to this basis are

$$
B_{m, n}=\frac{1}{\prod_{j=1}^{m} \sqrt{\beta_{j}}} \frac{1}{\prod_{k=1}^{n} \sqrt{\beta_{k}}} \int_{\mathbb{R}} p_{m}(x)(T+\gamma x) p_{n}(x) d \mu(x) .
$$

Clearly

$$
B_{m, n}=\left(\lambda_{n}+\gamma \alpha_{n}\right) \delta_{m, n}+\gamma \sqrt{\beta_{m}} \delta_{m, n+1}+\gamma \sqrt{\beta_{m+1}} \delta_{m, n-1} .
$$

The matrix $B=\left\{B_{m, n}\right\}$ is tridiagonal and generates the monic orthogonal polynomials via $\left\{\phi_{n}(E)\right\}$

$$
\begin{aligned}
& \phi_{0}(E)=1, \quad \phi_{1}(E)=E-\lambda_{0}-\gamma \alpha_{0}, \\
& \phi_{n+1}(E)=\left(E-\lambda_{n}-\gamma \alpha_{n}\right) \phi_{n}(E)-\gamma^{2} \beta_{n} \phi_{n-1}(E) .
\end{aligned}
$$


We can still scale $E$ by $E=\xi(x-\eta)$ and introduce additional parameters to help identify the polynomials as known ones. Thus we let $\psi_{n}(x)=\xi^{-n} \phi_{n}(E)$ and transform (5.4) to

$$
\begin{aligned}
& \psi_{0}(x)=1, \quad \psi_{1}(x)=x-\eta-\left(\lambda_{0}+\gamma \alpha_{0}\right) / \xi \\
& \psi_{n+1}(x)=\left[x-\eta-\left(\lambda_{n}+\gamma \alpha_{n}\right) / \xi\right] \psi_{n}(x)-(\gamma / \xi)^{2} \beta_{n} \psi_{n-1}(x) .
\end{aligned}
$$

The importance of this scaling will be made clear in the examples.

Recall that (5.2) generates a birth and death process polynomials if there are sequences $\left\{b_{n}\right\}$ and $\left\{d_{n}\right\}$ such that

$$
\alpha_{n}=b_{n}+d_{n}, \quad \beta_{n}=d_{n} b_{n-1},
$$

and for $n>0, b_{n-1}>0$ and $d_{n}>0$, with $d_{0} \geq 0$. One can represent the transition probability of going from state $m$ to state $n$ in time $t$ as the Laplace transform of the product of two orthogonal polynomials and their orthogonality measure. For details, and additional information and references see [21, Chapter 5] and the survey article [25].

The $b_{n}$ 's and $d_{n}$ 's are birth and death rates at state (population) $n$. In the case of birth and death processes with absorption (killing) Karlin and Tavaré [28] showed that the corresponding orthogonal polynomials satisfy (5.2) where (5.6) is now replaced by

$$
\alpha_{n}=b_{n}+d_{n}+c_{n}, \quad \beta_{n}=d_{n} b_{n-1},
$$

where $c_{n}$ is the absorption rate at state $n$. This leads to the following remark.

Remark 5.1. Assume that $T$ is a positive linear operator and (5.1) holds where $\left\{p_{n}\right\}$ are birth and death process polynomials with birth and death rates $\left\{b_{n}\right\}$ and $\left\{d_{n}\right\}$, respectively. Then the orthogonal polynomials in (5.5) with $\xi=\gamma$ which arise in the tridiagonalization of $T+\gamma x$ are polynomials associated with a birth and death process with absorption where the birth and death rates $\left\{b_{n}\right\}$ and $\left\{d_{n}\right\}$, respectively and the absorption rates are $\left\{\lambda_{n} / \gamma\right\}$.

The phenomena described in Remark 5.1 seem to be related to shape invariance and related topics in Discrete Quantum Mechanics recently developed by R. Sasaki and his coauthors, see the recent survey [35].

Example 5.2 (Laguerre polynomials). In this case $T$ is as on the left-hand side of (2.2) and

$$
\lambda_{n}=-n, \quad \alpha_{n}=2 n+\alpha+1, \quad \beta_{n}=n(n+\alpha), \quad p_{n}(x)=(-1)^{n} n ! L_{n}^{(\alpha)}(x) .
$$

The recursion in (5.5) is

$$
\psi_{n+1}(x)=\left[x-\eta+\frac{n(1-2 \gamma)}{\xi}-\frac{\gamma(\alpha+1)}{\xi}\right] \psi_{n}(x)-n(n+\alpha) \frac{\gamma^{2}}{\xi^{2}} \psi_{n-1}(x),
$$

with $E=\xi(x-\eta)$. When $\gamma=1 / 4$ we take $\xi=1 / 4, \eta=-2 \alpha-2$. This identifies $\psi_{n}(x)$ as $(-1)^{n} L_{n}^{(\alpha)}(x)$. Hence the spectrum is purely continuous and is given by $x \geq 0$, that is $E \geq(\alpha+1) / 2$. The absolutely continuous component is given by

$$
\mu^{\prime}(E)=4^{\alpha+1} \frac{\exp (2(\alpha+1))}{\Gamma(\alpha+1)}[E-(\alpha+1) / 2]^{\alpha} e^{-4 E}, \quad E \in[(\alpha+1) / 2, \infty) .
$$

We next assume $\gamma>1 / 4$ and compare (5.7) with the following monic form of (2.8)

$$
\psi_{n+1}(x)=[x-(n+\lambda) \cot \phi] \psi_{n}(x)-\frac{n(n+2 \lambda-1)}{4 \sin ^{2} \phi} \psi_{n-1}(x) .
$$


We make the parameter identification

$$
\gamma=\frac{1}{4} \sec ^{2}(\phi / 2), \quad \xi=-\tan (\phi / 2), \quad \lambda=(\alpha+1) / 2, \quad \eta=\frac{\alpha+1}{2} \cot (\phi / 2) .
$$

With this choice of parameters we identify the $\psi$ 's as Meixner-Pollaczek polynomials. Indeed $\psi_{n}(x)=P_{n}^{(\lambda)}(x ; \phi)$ where

$$
\gamma=\left(1+\xi^{2}\right) / 4, \quad \lambda=(\alpha+1) / 2, \quad \eta=-\frac{\alpha+1}{2 \xi},
$$

and

$$
\phi_{n}(E)=\xi^{-n} P_{n}^{(\lambda)}(\eta+E / \xi) .
$$

The spectral measure $\mu$ is absolutely continuous and when normalized to have a total mass 1 , its Radon-Nikodym derivative is

$$
\left(\frac{2 \xi}{1+\xi^{2}}\right)^{\alpha+1} \frac{\exp (2 \phi-\pi) x)}{\pi \xi \Gamma(\alpha+1)}|\Gamma(i x+(\alpha+1) / 2)|^{2} .
$$

We now consider the case $0<\gamma<1 / 4$. We identify (5.7) with the monic form of (2.6), namely

$$
y_{n+1}(x)=\left[x-\frac{n-c(\beta+n)}{1-c}\right] y_{n}(x)-\frac{c n(n+\beta-1)}{(1-c)^{2}} y_{n-1}(x) .
$$

This is done through the parameter identification

$$
\gamma=\frac{\sqrt{c}}{(1+\sqrt{c})^{2}}, \quad \xi=-\frac{1-\sqrt{c}}{1+\sqrt{c}}, \quad \beta=\alpha+1, \quad \eta=\frac{\beta \sqrt{c}}{1-\sqrt{c}} .
$$

It is clear from (2.6) that the $y_{n}$ 's are monic Meixner polynomials.

Note that such a division also occurs in the spectral decomposition of suitable elements in the Lie algebra $\mathfrak{s u}(1,1)$ in the discrete series representations, see [31, 33]. So one can ask for Lie algebraic interpretations along the lines of [34], see [36] for a related result.

Example 5.3 (Ultraspherical polynomials). In this case [21, (4.5.8)]

$$
T=\left(1-x^{2}\right)^{-\nu+1 / 2} \frac{d}{d x}\left(\left(1-x^{2}\right)^{\nu+1 / 2} \frac{d}{d x}\right), \quad \lambda_{n}=-n(n+2 \nu) .
$$

The coefficients in the monic form of the three term recurrence relation are, see $[29,(1.8 .18)]$,

$$
\alpha_{n}=0, \quad \beta_{n}=\frac{n(n+2 \nu-1)}{4(n+\nu)(n+\nu-1)} .
$$

Thus the recursion in (5.5) becomes

$$
\psi_{n+1}(x)=\left[x-\eta+\xi^{-1} n(n+2 \nu)\right] \psi_{n}(x)-\frac{\gamma^{2} \xi^{-2} n(n+2 \nu-1)}{4(n+\nu)(n+\nu-1)} \psi_{n-1}(x) .
$$

We do not know the orthogonality measure of the polynomials in (5.11). The special case $\nu=1$ appeared earlier in the work of Alhaidari and Bahlouli $[6,(3.8)]$ where they applied the $J$-matrix method to quantum model whose potential is an infinite potential well with sinusoidal bottom. The same case also appeared in the work [18] by Goh and Micchelli on certain aspects of the uncertainty principle. Determining the orthogonality measure of these polynomials will be very useful. 
The parameter $\eta$ can be absorbed in $x$, hence we assume $\eta=0$. In the notation of $(2.11)$

$$
b_{n}=-\frac{n(n+2 \nu)}{\xi}, \quad a_{n}^{2}=\frac{\gamma^{2} n(n+2 \nu-1)}{4 \xi^{2}(n+\nu)(n+\nu-1)} .
$$

In the case $\xi>0$, since $b_{n}<0$ and $b_{n}-b_{n-1}<0$, Theorem 2.3 implies that the smallest zero of $p_{n}(x)$ is approximately $\frac{1}{2}\left(b_{n}+b_{n-1}\right)-\frac{1}{2}\left|b_{n}-b_{n-1}\right|$. Hence

$$
x_{n, n}=-\xi^{-1} n(n+2 \nu)+\mathcal{O}(1) .
$$

On the other hand $a_{n}$ is monotone decreasing if $\nu \geq 1$ or $-1 / 2<\nu \leq 0$ and monotone increasing if $0<\nu \leq 1$. Using

$$
\begin{aligned}
\frac{1}{2}\left(b_{n}\right. & \left.+b_{n-1}\right) \pm \frac{1}{2} \sqrt{\left(b_{n}-b_{n-1}\right)^{2}+16 a_{n}^{2}} \\
& \leq \frac{1}{2}\left(b_{n}+b_{n-1}\right)+\frac{1}{2}\left|b_{n}-b_{n-1}\right|+2 a_{n} \leq 2 \max \left\{a_{1}, a_{\infty}\right\},
\end{aligned}
$$

where $a_{\infty}=\lim _{n \rightarrow \infty} a_{n}$. Therefore

$$
x_{n, 1}<2 \max \left\{a_{1}, a_{\infty}\right\} .
$$

Thus the spectrum is unbounded below and is contained in $\left(-\infty, 2 \max \left\{a_{1}, a_{\infty}\right\}\right)$. It is important to note that $p_{1}(0)=0$, hence the right end point of the spectrum, being $\lim _{n \rightarrow \infty} x_{n, 1}$ is positive. The case $\nu=1$ is the case when our starting point is the Chebyshev polynomials of the second kind. In this case $a_{n}=a_{1}$ for all $n$ and the largest zero of $p_{2}(x)$ is $-\frac{3}{2}+\sqrt{a_{1}^{2}+\frac{9}{4}}>0$.

Example 5.4 ( $q$-Ultraspherical polynomials). The weight function is supported on $[-1,1]$ and is given by $[21, \S 13.2]$

$$
w(x ; \beta) d x=\frac{\left(e^{2 i \theta}, e^{-2 i \theta} ; q\right)_{\infty}}{\left(\beta e^{2 i \theta}, \beta e^{-2 i \theta} ; q\right)_{\infty}} d \theta, \quad x=\cos \theta, \quad \beta<1 .
$$

The second order operator is $[21, \S 13.2]$

$$
T=\frac{1}{w(x ; \beta)} \mathcal{D}_{q}\left[w(x ; q \beta) \mathcal{D}_{q}\right], \quad \lambda_{n}=-\frac{4 q^{1-n}}{(1-q)^{2}}\left(1-q^{n}\right)\left(1-\beta^{2} q^{n}\right) .
$$

In this case

$$
\alpha_{n}=0, \quad \beta_{n}=\frac{\left(1-q^{n}\right)\left(1-\beta^{2} q^{n-1}\right)}{4\left(1-\beta q^{n}\right)\left(1-\beta q^{n-1}\right)},
$$

and the recurrence relation in (5.5) gives

$$
\begin{aligned}
\psi_{n+1}(x)= & {\left[x-\eta+\frac{4 q^{1-n} \xi^{-1}}{(1-q)^{2}}\left(1-q^{n}\right)\left(1-\beta^{2} q^{n}\right)\right] \psi_{n}(x) } \\
& -\frac{\gamma^{2}\left(1-q^{n}\right)\left(1-\beta^{2} q^{n-1}\right)}{4 \xi^{2}\left(1-\beta q^{n}\right)\left(1-\beta q^{n-1}\right)} \psi_{n-1}(x) .
\end{aligned}
$$

It is clear $\eta$ can be absorbed in $x$ so we may assume $\eta=0$. We do not know any explicit formulas for the above polynomials. It is clear that they are orthogonal on an unbounded set and that Condition (2.12) is satisfied, hence the orthogonality measure is unique. As in Example 5.3 
we can show the the spectrum is bounded above and unbounded below and estimate the largest and smallest zeros of $p_{n}(x)$. In the present case

$$
b_{n}=-\frac{4 q^{1-n} \xi^{-1}}{(1-q)^{2}}\left(1-q^{n}\right)\left(1-\beta^{2} q^{n}\right), \quad a_{n}^{2}=\frac{\gamma^{2}\left(1-q^{n}\right)\left(1-\beta^{2} q^{n-1}\right)}{4 \xi^{2}\left(1-\beta q^{n}\right)\left(1-\beta q^{n-1}\right)} .
$$

Here again the $b_{n}$ 's are negative and decreasing in $n$ for $\xi>0$. A simple calculation shows that $a_{n}$ increases with $n$ if $0<\beta<q$ and decreases with $n$ if $q<\beta<1$. Thus

$$
A:=\max \left\{a_{n}: n=1,2, \ldots\right\}= \begin{cases}a_{\infty} & \text { if } 0<\beta<q, \\ a_{1} & \text { if } q<\beta<1 .\end{cases}
$$

Therefore Theorem 2.3 shows that the smallest zero $x_{n, n}$ satisfies

$$
x_{n, n}>\frac{1}{2}\left(b_{n}+b_{n-1}\right)+\frac{1}{2}\left(b_{n}-b_{n-1}\right)-2 a_{n}=b_{n}-2 a_{n}>b_{n}-2 A .
$$

Indeed $x_{n, n}=b_{n}+\mathcal{O}(1)$. To determine the other end of the spectrum note that

$$
\sqrt{\left(b_{n}-b_{n-1}\right)^{2}+16 a_{n}^{2}}<\left|b_{n}-b_{n-1}\right|+4 a_{n}=b_{n-1}-b_{n}+4 a_{n} .
$$

Thus

$$
\begin{aligned}
\frac{1}{2}\left(b_{n}\right. & \left.+b_{n-1}\right)+\frac{1}{2} \sqrt{\left(b_{n}-b_{n-1}\right)^{2}+16 a_{n}^{2}} \\
& <\frac{1}{2}\left(b_{n}+b_{n-1}\right)+\frac{1}{2}\left(b_{n-1}-b_{n}\right)+2 a_{n}=b_{n-1}+2 a_{n} \leq 2 A, \quad n>0 .
\end{aligned}
$$

Consequently the largest zeros $x_{n, 1}$ is $<2 A$. Therefore the spectrum of $T+\gamma x$ is unbounded below and is contained in $(-\infty, 2 A]$.

Example 5.5 (Chebyshev polynomials). The Chebyshev polynomials of the first and second kinds are special ultraspherical polynomials and special $q$-ultraspherical polynomials as well. We will only discuss the polynomials $\left\{U_{n}(x)\right\}$ but the reader can easily write down the corresponding formulas for the polynomials $\left\{T_{n}(x)\right\}$.

The $U_{n}$ 's correspond to $\nu=1$ of (5.11) and the case $\beta=q$ of (5.12). Thus we are led to the following systems of orthogonal polynomials

$$
\begin{aligned}
& r_{n+1}(x)=\left[x-\eta+\xi^{-1} n(n+2)\right] r_{n}(x)-\frac{\gamma^{2}}{4 \xi^{2}} r_{n-1}(x), \\
& s_{n+1}(x)=\left[x-\eta+\frac{4 q^{1-n} \xi^{-1}}{(1-q)^{2}}\left(1-q^{n}\right)\left(1-q^{n+2}\right)\right] s_{n}(x)-\frac{\gamma^{2}}{4 \xi^{2}} s_{n-1}(x) .
\end{aligned}
$$

Here again we do not know any explicit representations or orthogonality measures for the polynomials $\left\{r_{n}(x)\right\}$ or $\left\{s_{n}(x)\right\}$. Again condition (2.12) is satisfied for $\left\{r_{n}(x)\right\}$ and $\left\{s_{n}(x)\right\}$. Therefore the orthogonality measures of both families of polynomials unique.

\section{Adding a linear potential}

In this section we yet have a variation on the problems of potential introduced at the beginning of Section 5. We start with (5.1) where the eigenfunctions satisfy (5.2). We then consider the Schrödinger operator

$$
S=(x+c) T+\gamma x \text {. }
$$


We illustrate this idea by considering the operators $T_{L}$ and $T_{M}$ for the Laguerre and Meixner polynomials defined in (3.1) and (4.2), respectively.

The Laguerre case. Here we take $c=0$ and

$$
T=\frac{e^{x}}{x^{\alpha+1}} \frac{d}{d x}\left[x^{\alpha+2} e^{-x} \frac{d}{d x}\right] .
$$

With the notation in (3.1) we have

$$
S=T_{L}+\gamma x
$$

We use the inner product (3.2) and our weighted $L_{2}$ space is $L_{2}\left(0, \infty, x^{\alpha} e^{-x}\right)$. The matrix elements $S_{m, n}$ are

$$
\begin{aligned}
& \sqrt{\frac{m ! n !}{\Gamma(\alpha+m+1) \Gamma(\alpha+n+1)}} \\
& \quad \times\left[\int_{0}^{\infty} L_{m}^{(\alpha)}(x) \frac{d}{d x}\left\{x^{\alpha+2} e^{-x} \frac{d}{d x} L_{n}^{(\alpha)}(x)\right\} d x+\gamma \int_{0}^{\infty} L_{m}^{(\alpha)}(x) x L_{n}^{(\alpha)}(x) x^{\alpha} e^{-x} d x\right] .
\end{aligned}
$$

Using the recurrence relation

$$
x L_{n}^{(\alpha)}(x)=-(n+1) L_{n+1}^{(\alpha)}(x)-(n+\alpha) L_{n-1}^{(\alpha)}(x)+(2 n+\alpha+1) L_{n}^{(\alpha)}(x),
$$

and the calculation of the matrix elements in (3.3) we find that

$$
\begin{aligned}
S_{m, n}= & {[\gamma(2 m+\alpha+1)-m(\alpha+2 m)] \delta_{m, n} } \\
& +(m-\gamma) \sqrt{(m+1)(m+\alpha+1)} \delta_{m, n-1}+(m-1-\gamma) \sqrt{m(m+\alpha)} \delta_{m, n+1} .
\end{aligned}
$$

The null space of $S$ is trivial so there is no need to mod out by the null space as we did in Sections 3 and 4 . The monic polynomials $\left\{p_{n}(E)\right\}$ which arise through tridiagonalization are generated by $p_{0}(E)=1, p_{1}(E)=E-\gamma(\alpha+1)$, and

$$
\begin{aligned}
E p_{n}(E)= & p_{n+1}(E)+n(n+\alpha)(n-1-\gamma)^{2} p_{n-1}(E) \\
& +[\gamma(2 n+\alpha+1)-n(\alpha+2 n)] p_{n}(E) .
\end{aligned}
$$

The polynomials in (6.2) form a two parameter subfamily of the continuous dual Hahn polynomials $[29, \S 1.3]$ with the parameters

$$
a=-\gamma-(\alpha+1) / 2, \quad b=c=(\alpha+1) / 2, \quad E=-x-\frac{1}{4}(\alpha+1)^{2} .
$$

In the above analysis we assumed $\alpha>-1$, hence $b=c>0$. If $\gamma<-(\alpha+1) / 2$ then $S$ has purely a continuous spectrum supported on $\left(-\infty,-(\alpha+1)^{2} / 4\right]$, see (3.4). If $\gamma>-(\alpha+1) / 2$ there is also discrete spectrum, and we obtain as in Section 3 the following proposition.

Proposition 6.1. The unbounded operator $S$ acting on the subspace of polynomials in $L_{2}\left(0, \infty, x^{\alpha} e^{-x}\right)$ is essentially self-adjoint. The spectrum of the closure has an absolutely continuous part $\left(-\infty,-\frac{1}{4}(\alpha+1)^{2}\right]$. The discrete spectrum consists of $\left\{E_{k} \mid k \in\{0, \ldots, M\}\right\}$, where $M=\max \{k \in \mathbb{N} \mid k-\gamma-(1+\alpha) / 2<0\}$ and $E_{k}=(k-\gamma)(k-\gamma-\alpha-1) \in\left(-\frac{1}{4}(\alpha+1)^{2}, \gamma(\gamma+\alpha+1)\right]$.

Remark 6.2. The case $\gamma=0$ of Proposition 6.1 reduces to Proposition 3.1 using a similar reduction as in [23, Remark 3.4]. Note that in this case $E_{0}=0$. 
Remark 6.3. In this case $S=T_{L}+\gamma x$ can be written as $S=x\left(D^{\alpha+1}+\gamma\right)$, where $D^{\alpha+1}$ is the second order differential operator

$$
D^{\alpha+1}=\frac{1}{x^{\alpha+1} e^{-x}} \frac{d}{d x}\left[x^{\alpha+2} e^{-x} \frac{d}{d x}\right],
$$

for which $D^{\alpha+1} L_{n}^{(\alpha+1)}(x)=-n L_{n}^{(\alpha+1)}(x)$. This shows that $S$ is of the type considered in [23].

The Meixner case. With the notation in (4.2) we let

$$
\tilde{S}=T_{M}+\frac{(1-c) \gamma}{c \beta(\beta+1)} x
$$

This corresponds to $c=0$ in (6.1), and $T_{M}$ as in (4.2). We use the inner product (4.3) and our space is now $L_{2}$ weighted with the orthogonality measure of the Meixner polynomials with parameters $c$ and $\beta$. The matrix elements $\tilde{S}_{m, n}$ are

$$
\begin{aligned}
& \sqrt{\frac{(\beta)_{m}(\beta)_{n}(1-c)^{2 \beta}}{c^{-m-n} m ! n !}}\left[\sum_{x=0}^{\infty} M_{m}(x ; \beta, c) \nabla\left(\frac{(\beta+2)_{x} c^{x}}{x !} \Delta M_{n}(x ; \beta, c)\right)\right. \\
& \left.+\frac{(1-c) \gamma}{c \beta(\beta+1)} \sum_{x=0}^{\infty} \frac{c^{x}(\beta)_{x}}{x !} M_{m}(x ; \beta, c) x M_{n}(x ; \beta, c)\right] .
\end{aligned}
$$

We already calculated the matrix elements of $T_{M}$ in (4.4), but we must replace $m, n$ by $m-1$, $n-1$, respectively. Using the recurrence relation (2.6) we then compute the matrix elements of a constant times $x$. This leads to

$$
\begin{aligned}
& c \beta(\beta+1) \tilde{S}_{m, n}=-[m(m+\beta)+m(m-1) c+\gamma m+c \gamma(\beta+m)] \delta_{m, n} \\
& \quad+(m-1-\gamma) \sqrt{c m(m+\beta-1)} \delta_{m, n+1}+(m-\gamma) \sqrt{c(m+1)(\beta+m)} \delta_{m, n-1} .
\end{aligned}
$$

Therefore the corresponding orthonormal polynomials $p_{n}(E)$ satisfy the three term recurrence relation

$$
\begin{aligned}
& c \beta(\beta+1) E p_{m}(E)=-[m(m+\beta)+m(m-1) c+\gamma m+c \gamma(\beta+m)] p_{m}(E) \\
& \quad+(m-\gamma) \sqrt{c(m+1)(\beta+m)} p_{m+1}(E)+(m-1-\gamma) \sqrt{c m(m+\beta-1)} p_{m-1}(E) .
\end{aligned}
$$

We assume $0<c<1, \beta>0$, since we are dealing with the Meixner polynomials. In order to have (6.3) satisfy the conditions for an orthonormal polynomial system we assume $\gamma<0$. The polynomials generated by (6.3) seem to be new. Here again we have neither explicit representations or generating functions, nor do we know their orthogonality measure. One can say however that their orthogonality measure is unique since condition (2.13) is clearly satisfied for sufficiently large $n$. Using Theorem 2.3 and some estimates we see that the support of the orthogonality measure is contained in $(-\infty, a]$ for some $a>0$.

\section{The Al-Salam-Chihara polynomials}

Recall that $w$ is defined in (2.9). The generating function (2.10) implies

$$
\left(1-t_{1} t_{2}\right) p_{n}\left(x ; t_{1}, t_{2}\right)=\left(1-t_{1} t_{2} q^{n}\right) p_{n}\left(x ; t_{1}, q t_{2}\right)-t_{1} t_{2}\left(1-q^{n}\right) p_{n-1}\left(x ; t_{1}, q t_{2}\right) .
$$

We will first consider the case when the operator $T$ is

$$
L:=\frac{1}{w\left(x ; t_{1}, t_{2}\right)} \mathcal{D}_{q}\left[w\left(x ; q^{1 / 2} t_{1}, q^{3 / 2} t_{2}\right) \mathcal{D}_{q}\right] .
$$


Apply (15.1.6) and (12.2.2) in [21] to see that

$$
\mathcal{D}_{q} p_{n}\left(x ; t_{1}, t_{2}\right)=\frac{2 t_{1} q^{1-n}\left(1-q^{n}\right)}{(1-q)\left(1-t_{1} t_{2}\right)} p_{n-1}\left(x ; t_{1} \sqrt{q}, t_{2} \sqrt{q}\right) .
$$

Moreover

$$
\mathcal{D}_{q}\left[w\left(x, \sqrt{q} t_{1}, \sqrt{q} t_{2}\right) p_{n-1}\left(x ; \sqrt{q} t_{1}, \sqrt{q} t_{2}\right)\right]=\frac{2\left(1-t_{1} t_{2}\right)}{t_{1}(q-1)} w\left(x, t_{1}, t_{2}\right) p_{n}\left(x ; t_{1}, t_{2}\right) .
$$

For real $t_{1}, t_{2}$ with $\left|t_{1}\right|,\left|t_{2}\right|<1$ the Al-Salam-Chihara polynomials $\left\{p_{n}\left(x ; t_{1}, t_{2}\right)\right\}$ satisfy the orthogonality relation $[21,(15.1 .5)]$,

$$
\begin{aligned}
& h_{n}\left(t_{1}, t_{2}\right) \delta_{m, n}=\int_{-1}^{1} p_{m}\left(x ; t_{1}, t_{2}\right) p_{n}\left(x ; t_{1}, t_{2}\right) w\left(x ; t_{1}, t_{2}\right) d x, \\
& h_{n}\left(t_{1}, t_{2}\right)=\frac{2 \pi(q ; q)_{n} t_{1}^{2 n}}{\left(q, t_{1} t_{2} ; q\right)_{\infty}\left(t_{1} t_{2} ; q\right)_{n}},
\end{aligned}
$$

and are complete in $L^{2}\left(-1,1 ; w\left(x ; t_{1}, t_{2}\right) d x\right)$. In view of (7.4) the orthonormal Al-Salam-Chihara polynomials are

$$
\tilde{p}_{n}\left(x ; t_{1}, t_{2}\right)=\sqrt{\frac{\left(q, t_{1} t_{2} ; q\right)_{\infty}\left(t_{1} t_{2} ; q\right)_{n}}{2 \pi t_{1}^{2 n}(q ; q)_{n}}} p_{n}\left(x ; t_{1}, t_{2}\right) .
$$

Lemma 7.1. Let $\left\{A_{m, n}(A C)\right\}$ be the matrix elements of $L$ in the basis $\left\{\tilde{p}_{n}\left(x ; t_{1}, t_{2}\right)\right\}$. Then

$$
\begin{aligned}
A_{m, n}= & -\frac{4 q^{1-m}\left(1-q^{m}\right)}{(1-q)^{2}}\left[1-t_{1} t_{2} q^{m}+t_{2}^{2}\left(q-q^{m}\right)\right] \delta_{m, n} \\
& +\frac{4 t_{2} q^{1-m}\left(1-q^{m}\right)}{(1-q)^{2}} \sqrt{\left(1-q^{m+1}\right)\left(1-t_{1} t_{2} q^{m}\right)} \delta_{m, n-1} \\
& +\frac{4 t_{2} q^{2-m}\left(1-q^{m-1}\right)}{(1-q)^{2}} \sqrt{\left(1-q^{m}\right)\left(1-t_{1} t_{2} q^{m-1}\right)} \delta_{m, n+1} .
\end{aligned}
$$

Proof. Clearly equation (7.2) implies

$$
\begin{aligned}
& \int_{-1}^{1} p_{m}\left(x ; t_{1}, t_{2}\right) w\left(x ; t_{1}, t_{2}\right) L p_{n}\left(x ; t_{1}, t_{2}\right) d x \\
& \quad=\frac{2 t_{1}\left(1-q^{n}\right) q^{1-n}}{(1-q)\left(1-t_{1} t_{2}\right)} \int_{-1}^{1} p_{m}\left(x ; t_{1}, t_{2}\right) \mathcal{D}_{q}\left[w\left(x ; q^{1 / 2} t_{1}, q^{3 / 2} t_{2}\right) p_{n-1}\left(x ; t_{1} \sqrt{q}, t_{2} \sqrt{q}\right)\right] d x .
\end{aligned}
$$

In view of (7.1) the integrand in the last step is

$$
\begin{aligned}
& {\left[\frac{\left(1-t_{1} t_{2} q^{m}\right)}{1-t_{1} t_{2}} p_{m}\left(x ; t_{1}, q t_{2}\right)-\frac{t_{1} t_{2}\left(1-q^{m}\right)}{1-t_{1} t_{2}} p_{m-1}\left(x ; t_{1}, q t_{2}\right)\right]} \\
& \quad \times \mathcal{D}_{q}\left[w ( x ; q ^ { 1 / 2 } t _ { 1 } , q ^ { 3 / 2 } t _ { 2 } ) \left\{\frac{\left(1-t_{1} t_{2} q^{n}\right)}{1-q t_{1} t_{2}} p_{n-1}\left(x ; \sqrt{q} t_{1}, q^{3 / 2} t_{2}\right)\right.\right. \\
& \left.\left.\quad-\frac{t_{1} t_{2}\left(q-q^{n}\right)}{1-q t_{1} t_{2}} p_{n-2}\left(x ; \sqrt{q} t_{1}, q^{3 / 2} t_{2}\right)\right\}\right] .
\end{aligned}
$$

Applying (7.3) we see that the quantity after the $\times$ is

$$
\frac{2}{t_{1}(q-1)} w\left(x ; t_{1}, q t_{2}\right)\left[\left(1-t_{1} t_{2} q^{n}\right) p_{n}\left(x ; t_{1}, q t_{2}\right)-t_{1} t_{2}\left(q-q^{n}\right) p_{n-1}\left(x ; t_{1}, q t_{2}\right)\right] .
$$


Therefore

$$
\begin{aligned}
&-\frac{(1-q)^{2}\left(1-t_{1} t_{2}\right)^{2}}{4\left(1-q^{n}\right) q^{1-n}} \int_{-1}^{1} p_{m}\left(x ; t_{1}, t_{2}\right) w\left(x ; t_{1}, t_{2}\right) L p_{n}\left(x ; t_{1}, t_{2}\right) d x \\
&=\int_{-1}^{1} w\left(x, t_{1}, q t_{2}\right)\left[\left(1-t_{1} t_{2} q^{m}\right) p_{m}\left(x ; t_{1}, q t_{2}\right)-t_{1} t_{2}\left(1-q^{m}\right) p_{m-1}\left(x ; t_{1}, q t_{2}\right)\right] \\
& \quad \times\left[\left(1-t_{1} t_{2} q^{n}\right) p_{n}\left(x ; t_{1}, q t_{2}\right)-t_{1} t_{2}\left(q-q^{n}\right) p_{n-1}\left(x ; t_{1}, q t_{2}\right)\right] d x \\
&=\left[\left(1-t_{1} t_{2} q^{m}\right)^{2} h_{m}\left(t_{1}, q t_{2}\right)+t_{1}^{2} t_{2}^{2}\left(1-q^{m}\right)\left(q-q^{m}\right) h_{m-1}\left(t_{1}, q t_{2}\right)\right] \delta_{m, n} \\
&-q t_{1} t_{2}\left(1-q^{m}\right)\left(1-t_{1} t_{2} q^{m}\right) h_{m}\left(t_{1}, q t_{2}\right) \delta_{m, n-1} \\
&-t_{1} t_{2}\left(1-q^{m}\right)\left(1-t_{1} t_{2} q^{m-1}\right) h_{m-1}\left(t_{1}, q t_{2}\right) \delta_{m, n+1} .
\end{aligned}
$$

The result follows since

$$
A_{m, n}=\frac{1}{\sqrt{h_{m}\left(t_{1}, t_{2}\right) h_{n}\left(t_{1}, t_{2}\right)}} \int_{-1}^{1} p_{m}\left(x ; t_{1}, t_{2}\right) w\left(x ; t_{1}, t_{2}\right) L p_{n}\left(x ; t_{1}, t_{2}\right) d x .
$$

The monic orthogonal polynomials generated by the matrix $\frac{4}{(1-q)^{2}} A$, with $A=\left\{A_{m, n}(A C)\right\}$ satisfy

$$
\begin{aligned}
P_{n+1}(x)= & {\left[x-q^{-n}\left(1-q^{n+1}\right)\left[1-t_{1} t_{2} q^{n+1}+t_{2}^{2} q\left(1-q^{n}\right)\right] P_{n}(x)\right.} \\
& -t_{2}^{2} q^{2-2 n}\left(1-q^{n+1}\right)\left(1-q^{n}\right)^{2}\left(1-t_{1} t_{2} q^{n}\right) P_{n-1}(x) .
\end{aligned}
$$

Note that the recurrence relation (7.5) is invariant under $q \rightarrow 1 / q$ after scaling and renaming the parameters. The recurrence coefficients grow exponentially, and by [11, Theorem VII.1.5] we can easily check that the moment problem corresponding problem does not have a unique solution (indeterminate). Hence $L$ with domain the polynomials is not essentially self-adjoint. Nothing is known about the explicit formulas of the polynomials generated by (7.5) or any of their orthogonality measures.

The $\boldsymbol{q}^{\mathbf{- 1}}$-Hermite polynomials. We now study the $q^{-1}$-Hermite polynomials of Askey [9], Ismail and Masson [27]. They are generated by $h_{0}(x \mid q), h_{1}(x \mid q)=2 x$, and

$$
h_{n+1}(x \mid q)=2 x h_{n}(x \mid q)-q^{-n}\left(1-q^{n}\right) h_{n-1}(x \mid q) .
$$

Here we use the parametrization $x=\sinh \xi$. Recall the definitions [21, Chapter 21], [22]

$$
\begin{aligned}
& f(x)=\breve{f}\left(e^{\xi}\right), \quad\left(\mathcal{D}_{q} f\right):=\frac{\breve{f}\left(q^{1 / 2} e^{\xi}\right)-\breve{f}\left(q^{-1 / 2} e^{\xi}\right)}{\left(q^{1 / 2}-q^{-1 / 2}\right)\left(e^{\xi}+e^{-\xi}\right) / 2}, \\
& \left(\mathcal{A}_{q} f\right)(x)=\frac{1}{2}\left[\breve{f}\left(q^{1 / 2} e^{\xi}\right)+\breve{f}\left(q^{-1 / 2} e^{\xi}\right)\right] .
\end{aligned}
$$

The corresponding moment problem is indeterminate but all the $N$-extremal measures have been determined in [27], see also $[13, \S 4]$ for another proof. They are purely discrete and are enumerated by a parameter $a \in(q, 1)$. The support is $\left\{x_{n}(a): n=0, \pm 1, \pm 2, \ldots\right\}$ and

$$
x_{n}(a)=\frac{1}{2}\left[q^{-n} / a-a q^{n}\right], \quad \mu\left(\left\{x_{n}(a)\right\}\right)=\frac{a^{4 n} q^{n(2 n-1)}\left(1+a^{2} q^{2 n}\right)}{\left(-a^{2},-q / a^{2}, q ; q\right)_{\infty}},
$$

where $\mu$ is the corresponding normalized orthogonality measure. The orthogonality relation is

$$
\int_{\mathbb{R}} h_{m}(x \mid q) h_{n}(x \mid q) d \mu(x)=q^{-n(n+1) / 2}(q ; q)_{n} \delta_{m, n} .
$$


The lowering operator is

$$
\mathcal{D}_{q} h_{n}(x \mid q)=\frac{2\left(1-q^{n}\right)}{1-q} q^{(1-n) / 2} h_{n-1}(x \mid q) .
$$

The second order operator equation satisfied by the $q^{-1}$-Hermite polynomials is [22]

$$
q^{1 / 2}\left(1+2 x^{2}\right) \mathcal{D}_{q}^{2} y+\frac{4 q}{q-1} x \mathcal{A}_{q} \mathcal{D}_{q} y=\lambda_{n} y, \quad \lambda_{n}:=-\frac{4 q\left(1-q^{n}\right)}{(1-q)^{2}} .
$$

With the measure $\mu$ defined as in (7.6) the matrix elements of the operator $T_{H}+\gamma x$ with $T_{H}$ the operator on the left side of $(7.7)$ on $L_{2}(\mathbb{R}, \mu)$ with basis $\left\{\tilde{h}_{n}=q^{n(n+1) / 4} h_{n}(x \mid q) / \sqrt{(q ; q)_{n}}\right\}$ are given by

$$
\left(T_{H}+\gamma x\right) \tilde{h}_{n}=\frac{\gamma}{2} q^{-(n+1) / 2} \sqrt{1-q^{n+1}} \tilde{h}_{n+1}+\lambda_{n} \tilde{h}_{n}+\frac{\gamma}{2} q^{-n / 2} \sqrt{1-q^{n}} \tilde{h}_{n-1} .
$$

Therefore the corresponding monic polynomials $\left\{p_{n}(E)\right\}$ are generated by

$$
E p_{n}(E)=p_{n+1}(E)-\frac{4 q\left(1-q^{n}\right)}{(1-q)^{2}} p_{n}(E)+\frac{\gamma^{2}}{4} q^{-n}\left(1-q^{n}\right) p_{n-1}(E) .
$$

This is essentially a perturbation of the Jacobi matrix of the $q^{-1}$-Hermite polynomials by the diagonal matrix $-\frac{4 q\left(1-q^{n}\right)}{(1-q)^{2}}$. Apart from the shift $\frac{-4 q}{(1-q)^{2}} \mathrm{Id}$, this is a compact perturbation of the Jacobi matrix for the $q^{-1}$-Hermite polynomials. Using [11, Chapter VII, Theorem 1.5] the moment problem corresponding to the orthogonal polynomials generated by (7.8) is indeterminate.

We now give bounds for the zeros of $p_{n}(E)$. In the present case

$$
a_{n}^{2}=\frac{\gamma^{2}}{4} q^{-n}\left(1-q^{n}\right), \quad b_{n}=-\frac{4 q\left(1-q^{n}\right)}{(1-q)^{2}} .
$$

Take $\gamma>0$, it is clear that $a_{n}$ is monotonic increasing while $b_{n}$ is monotic decreasing. The use of

$$
\sqrt{\left(b_{n}-b_{n-1}\right)^{2}+16 a_{n}^{2}}<\left|b_{n}-b_{n-1}\right|+4 a_{n},
$$

shows that the $x_{j}$ 's and $y_{j}$ 's in (2.15) satisfy

$$
\begin{aligned}
x_{j} & <\frac{1}{2}\left(b_{j}+b_{j-1}\right)+\frac{1}{2}\left(b_{j-1}-b_{j}\right)+2 a_{j}=b_{j-1}+2 a_{j}<2 a_{n}, \\
y_{j} & >\frac{1}{2}\left(b_{j}+b_{j-1}\right)-\frac{1}{2}\left(b_{j-1}-b_{j}\right)-2 a_{j}=b_{j}-2 a_{j} \geq-2 a_{n}-\frac{4 q}{(1-q)^{2}} .
\end{aligned}
$$

Therefore

$$
x_{n, 1}<\gamma q^{-n / 2} \sqrt{1-q^{n}}, \quad x_{n, n}>-\gamma q^{-n / 2} \sqrt{1-q^{n}}-\frac{4 q}{(1-q)^{2}} .
$$

\section{Acknowledgements}

The research of Mourad E.H. Ismail is supported by a Research Grants Council of Hong Kong under contract \# 101411 and NPST Program of King Saud University, Saudi Arabia, 10-MAT 1293-02. This work was also partially supported by a grant from the 'Collaboration Hong Kong Joint Research Scheme' sponsored by the Netherlands Organisation of Scientific Research and the Research Grants Council for Hong Kong (Reference number: 600.649.000.10N007). The work for this paper was done while both authors visited City University Hong Kong, and we are grateful for the hospitality.

We thank Luc Vinet and Hocine Bahlouli for useful comments and references. We also thank the referees for their very careful reading and for their suggestions and constructive criticism that have improved the paper. 


\section{References}

[1] Akhiezer N.I., The classical moment problem and some related questions in analysis, Hafner Publishing Co., New York, 1965.

[2] Alhaidari A.D., An extended class of $L^{2}$-series solutions of the wave equation, Ann. Physics 317 (2005), 152-174, quant-ph/0409002.

[3] Alhaidari A.D., Exact $L^{2}$ series solution of the Dirac-Coulomb problem for all energies, Ann. Physics 312 (2004), 144-160, hep-th/0405023.

[4] Alhaidari A.D., Group-theoretical foundation of the J-matrix theory of scattering, J. Phys. A: Math. Gen. 33 (2000), 6721-6738.

[5] Alhaidari A.D., $L^{2}$ series solution of the relativistic Dirac-Morse problem for all energies, Phys. Lett. A 326 (2004), 58-69, math-ph/0405008.

[6] Alhaidari A.D., Bahlouli H., Extending the class of solvable potentials. I. The infinite potential well with a sinusoidal bottom, J. Math. Phys. 49 (2008), 082102, 13 pages.

[7] Alhaidari A.D., Bahlouli H., Abdelmonem M.S., Al-Ameen F., Al-Abdulaal T., Regularization in the $J$ matrix method of scattering revisited, Phys. Lett. A 364 (2007), 372-377.

[8] Andrews G.E., Askey R., Roy R., Special functions, Encyclopedia of Mathematics and its Applications, Vol. 71, Cambridge University Press, Cambridge, 1999.

[9] Askey R., Continuous $q$-Hermite polynomials when $q>1$, in $q$-Series and Partitions (Minneapolis, MN, 1988), IMA Vol. Math. Appl., Vol. 18, Springer, New York, 1989, 151-158.

[10] Bastard G., Wave mechanics applied to semiconductor heterostructures, Wiley-Interscience, 1991.

[11] Berezans'kiü J.M., Expansions in eigenfunctions of selfadjoint operators, Translations of Mathematical Monographs, Vol. 17, American Mathematical Society, Providence, R.I., 1968.

[12] Brown B.M., Evans W.D., Ismail M.E.H., The Askey-Wilson polynomials and q-Sturm-Liouville problems, Math. Proc. Cambridge Philos. Soc. 119 (1996), 1-16, math.CA/9408209.

[13] Christiansen J.S., Koelink E., Self-adjoint difference operators and symmetric Al-Salam-Chihara polynomials, Constr. Approx. 28 (2008), 199-218, math.CA/0610534.

[14] Deift P.A., Orthogonal polynomials and random matrices: a Riemann-Hilbert approach, Courant Lecture Notes in Mathematics, Vol. 3, New York University Courant Institute of Mathematical Sciences, New York, 1999.

[15] Edmunds D.E., Evans W.D., Spectral theory and differential operators, Oxford Mathematical Monographs, Oxford Science Publications, The Clarendon Press, Oxford University Press, New York, 1987.

[16] Erdélyi A., Magnus W., Oberhettinger F., Tricomi F.G., Higher transcendental functions, Vol. 1, McGrawHill, 1953.

[17] Gasper G., Rahman M., Basic hypergeometric series, Encyclopedia of Mathematics and its Applications, Vol. 96, 2nd ed., Cambridge University Press, Cambridge, 2004.

[18] Goh S.S., Micchelli C.A., Uncertainty principles in Hilbert spaces, J. Fourier Anal. Appl. 8 (2002), 335-373.

[19] Heller E.J., Theory of J-matrix Green's functions with applications to atomic polarizability and phase-shift error bounds, Phys. Rev. A 12 (1975), 1222-1231.

[20] Heller E.J., Reinhardt W.P., Yamani H.A., On an "equivalent quadrature" calculation of matrix elements of $\left(z-P^{2} / 2 m\right)$ using an $L^{2}$ expansion technique, J. Comp. Phys. 13 (1973), 536-549.

[21] Ismail M.E.H., Classical and quantum orthogonal polynomials in one variable, Encyclopedia of Mathematics and its Applications, Vol. 98, Cambridge University Press, Cambridge, 2009.

[22] Ismail M.E.H., Ladder operators for $q^{-1}$-Hermite polynomials, C.R. Math. Rep. Acad. Sci. Canada 15 (1993), 261-266.

[23] Ismail M.E.H., Koelink E., Spectral properties of operators using tridiagonalization, Anal. Appl. 10 (2012), 327-343, arXiv:1108.5716.

[24] Ismail M.E.H., Koelink E., The J-matrix method, Adv. in Appl. Math. 46 (2011), 379-395, arXiv:0810.4558.

[25] Ismail M.E.H., Letessier J., Masson D., Valent G., Birth and death processes and orthogonal polynomials, in Orthogonal Polynomials: Theory and Practice (Columbus, OH, 1989), NATO Advanced Science Institutes Series C: Mathematical and Physical Sciences, Vol. 294, Editor P. Nevai, Kluwer Acad. Publ., Dordrecht, 1990, 225-229. 
[26] Ismail M.E.H., Li X., Bound on the extreme zeros of orthogonal polynomials, Proc. Amer. Math. Soc. 115 (1992), 131-140.

[27] Ismail M.E.H., Masson D.R., $q$-Hermite polynomials, biorthogonal rational functions, and $q$-beta integrals, Trans. Amer. Math. Soc. 346 (1994), 63-116.

[28] Karlin S., Tavaré S., Linear birth and death processes with killing, J. Appl. Probab. 19 (1982), $477-487$.

[29] Koekoek R., Swarttouw R.F., The Askey-scheme of hypergeometric orthogonal polynomials and its $q$ analogue, Report 98-17, Faculty of Technical Mathematics and Informatics, Delft University of Technology, 1998, http://aw.twi.tudelft.nl/ koekoek/askey/.

[30] Koelink E., Spectral theory and special functions, in Laredo Lectures on Orthogonal Polynomials and Special Functions, Adv. Theory Spec. Funct. Orthogonal Polynomials, Nova Sci. Publ., Hauppauge, NY, 2004, 45-84, math.CA/0107036.

[31] Koelink H.T., Van der Jeugt J., Convolutions for orthogonal polynomials from Lie and quantum algebra representations, SIAM J. Math. Anal. 29 (1998), 794-822, q-alg/9607010.

[32] Landau L.D., Lifshitz L.M., Course of theoretical physics, Vol. 3, Quantum mechanics: nonrelativistic theory, 3rd ed., Butterworth-Heinemann, 1991.

[33] Masson D.R., Repka J., Spectral theory of Jacobi matrices in $l^{2}(\mathbf{Z})$ and the su(1,1) Lie algebra, SIAM J. Math. Anal. 22 (1991), 1131-1146.

[34] Miller Jr. W., Lie theory and special functions, Mathematics in Science and Engineering, Vol. 43, Academic Press, New York, 1968.

[35] Odake S., Sasaki R., Discrete quantum mechanics, J. Phys. A: Math. Theor. 44 (2011), 353001, 47 pages, arXiv:1104.0473.

[36] Ojha P.C., SO $(2,1)$ Lie algebra, the Jacobi matrix and the scattering states of the Morse oscillator, J. Phys. A: Math. Gen. 21 (1988), 875-883.

[37] Rainville E.D., Special functions, The Macmillan Co., New York, 1960.

[38] Simon B., The classical moment problem as a self-adjoint finite difference operator, Adv. Math. 137 (1998), 82-203, math-ph/9906008.

[39] Szegö G., Orthogonal polynomials, American Mathematical Society, Colloquium Publications, Vol. 23, 4th ed., American Mathematical Society, Providence, R.I., 1975.

[40] Yamani H.A., Reinhardt W.P., $L^{2}$ discretizations of the continuum: radial kinetic energy, Phys. Rev. A 11 (1975), 1144-1156. 